\title{
Longitudinal investigation of severe acute respiratory syndrome coronavirus 2 (SARS-CoV-2) infection in older patients in the province of Palermo (Southern Italy) during the early wave of the pandemic
}

\author{
Domenica Matranga ${ }^{1}$, Laura Maniscalco², Marco Enea ${ }^{1}$, Dario De Luca³, Davide Brancato ${ }^{3}$, \\ Emanuele La Spada ${ }^{3}$, Alessandro Scorsone ${ }^{3}$, Paola Di Carlo ${ }^{1}$
}

\begin{abstract}
${ }^{1}$ Department of Health Promotion, Mother and Child Care, Internal Medicine and Medical Specialties, University of Palermo, Palermo, Italy

${ }^{2}$ Department of Biomedicine, Neuroscience and Advanced Diagnostics, University of Palermo, Palermo, Italy

${ }^{3}$ Partinico Covid Hospital, Azienda Sanitaria Provinciale di Palermo, Palermo, Italy
\end{abstract}

Submitted: 17 November 2020; Accepted: 6 March 2021

Online publication: 18 March 2021

Arch Med Sci 2022; 18 (6): 1488-1497

DOI: https://doi.org/10.5114/aoms/134024

Copyright (c) 2021 Termedia \& Banach

\begin{abstract}
Introduction: Clinical presentation of severe acute respiratory syndrome coronavirus 2 (SARS-CoV-2) infection in old adults from Southern Italy is little known. This study aims to investigate the mortality risk related to risk factors, therapy and clinical course and to suggest prognostic indicators based on day-to-day follow-up of clinical and laboratory findings.

Material and methods: It was designed as a retrospective longitudinal cohort study of adult SARS-CoV-2 patients admitted at Partinico COVID Hospital in Palermo, Southern Italy. Patients were recruited between 4 March and 25 April and followed up until 31 May 2020, day-to-day until death or hospital discharge. Clinical data, laboratory tests and treatment data were extracted from medical records and epidemiologic information was obtained by clinical history and the medical interview.

Results: Forty-seven patients (median age $=75$ IQR: 59.50-86.00) were followed up during a 87 days observation period, accounting for a total of 1,035 person days. At the end of follow-up, 28 (60\%) patients were discharged and 19 (40\%) died, so that the estimated incidence density rate was 0.018 deaths per day (18 SARS-CoV-2-related deaths per 1,000 patient days). Diabetes $(H R=8.13,95 \% \mathrm{Cl}: 1.91-34.67)$, chronic kidney failure $(H R=5.86$, $95 \% \mathrm{Cl}: 1.36-25.21)$, dementia $(\mathrm{HR}=7.84,95 \% \mathrm{Cl}: 1.80-34.20)$, and neutrophil/lymphocyte ratio $>7(\mathrm{HR}=10.37,95 \% \mathrm{Cl}: 2.24-48.14)$ were found as significant prognostic factors.

Conclusions: The joint evaluation of dementia, diabetes, chronic kidney failure and neutrophil/lymphocyte ratio showed an optimal prognostic value already in the first week of follow-up. The day-to-day follow-up provides essential information for clinical monitoring and treatment of the disease in a hospital setting and improves the disease's home management, especially for older patients with frailty.
\end{abstract}

Key words: dementia, diabetes, prognostic factors, chronic kidney failure, D-dimer, neutrophil/lymphocyte ratio.

\section{Corresponding author:}

Prof. Domenica Matranga

Department of

Health Promotion,

Mother and Child Care

Internal Medicine and

Medical Specialties University

Palermo, Italy

E-mail: domenica.matranga@

unipa.it 


\section{Introduction}

In the early wave of the severe acute respiratory syndrome coronavirus 2 (SARS-CoV-2) epidemic, Italy reported a higher number of recorded cases and deaths than other European countries. By 31 May 2020, a total of 232,639 confirmed cases and 32,981 deaths had been reported, indicating a standardized mortality rate of 46.8 per 100,000 inhabitants [1].

The clinical presentation of the disease at the outbreak of the pandemic was very heterogeneous nationwide. In Northern Italy, the disease spectrum showed more severe signs characterized by a high fatality rate, frequent admissions at intensive care units (ICUs), older patients and a higher number of comorbidities [2, 3]. Conversely, the early few epidemiologic studies in Southern Italy $[4,5]$ suggested that the infection spread at a lower rate and less severely than in Northern Italian Regions [6, 7]. The heterogeneous distribution of SARS-CoV-2 infection across Italian regions could be related to different environmental conditions, such as the past and cumulative exposure to particulate matter pollution [8].

Emerging evidence shows that older people [9] and people with multimorbidity present a more severe disease spectrum, in both cases of clinical assessment based on patients' clinical symptoms, signs, and chest imaging manifestations [10] and in case of occurrence of ICU admission and death [11]. It has been documented that elderly patients have the highest mortality rates [9], show a significantly higher risk of developing acute respiratory distress syndrome (ARDS) [12] and progressing towards death $[3,13]$.

The majority of primary studies concerning the clinical characteristics and prognosis of patients with SARS-COV-2 are retrospective cohorts, with clinical information only evaluated at the baseline [14-16] or used as a stratification variable of the survival experience of the cohort [17]. Only a few hospital-based studies take full advantage of the cohort design by tracking patients' clinical and laboratory parameters from admission to discharge. Some of these studies consider the D-dimer trend [18], the temporal evolution of C-reactive protein concentration and lymphocyte count [19], the variation of haematological and immunologic biomarkers in patients with SARS-COV-2 infection across three time periods [20], the clinical courses of major symptoms, the outcomes and the viral shedding [21].

With this retrospective longitudinal cohort study, we propose to fill in two knowledge shortfalls:

- to generate evidence about the mortality risk for SARS-COV-2 disease, relating to epidemiological and clinical factors, therapy and clinical course, in a sample of elderly patients during the early wave of the pandemic across Southern Italy,

- to suggest prognostic indicators based on the day-to-day follow-up of clinical and laboratory findings.

\section{Material and methods}

\section{Study design and participants}

We performed a retrospective longitudinal cohort study of all patients admitted for SARS-CoV-2 infection at Partinico COVID Hospital in the province of Palermo (Sicily, Southern Italy) between 4 March and 25 April and followed up until 31 May 2020. Patients referred to this centre came from the emergency departments of other hospitals in Palermo with a confirmed diagnosis of SARSCoV-2 infection. Diagnosis of SARS-COV-2 was confirmed by a positive real-time reverse transcriptase PCR from nasal swabs and analysed by the molecular virology unit according to the WHO guidelines and protocol by Corman et al. [22]. Patients were followed up day-to-day until death or hospital discharge. The criterion for discharge was the occurrence of two consecutive nasal and pharyngeal swabs, at an interval of at least $24 \mathrm{~h}$, negative for SARS-CoV-2 RNA.

A multidisciplinary team made of an infectious physician, a pulmonologist, a cardiologist, a diabetes specialist, a haematologist, an anaesthesiologist and an internist was responsible for the clinical management of SARS-COV-2 patients. Psychiatric and psychological support was available for hospitalized patients and the working medical team.

\section{Ethical statement}

The research was conducted according to the Helsinki Declaration. All patients signed the informed consent upon admission to the hospital. The study protocol received the ethical approval from the local Ethical Committee (Azienda Ospedaliera Policlinico "Paolo Giaccone" of Palermo, No. 4/2020, dated 22 April 2020).

\section{Data collection}

We collected demographic data (sex and age), epidemiological information, clinical data, laboratory tests and treatment data. The data were extracted from medical records, and the epidemiologic information was obtained by the clinical history and by the medical interview carried out during patients' hospitalization.

The epidemiological information included i) recent exposure to people with confirmed SARSCoV-2 infection, ii) coming from a recent journey abroad or in other Italian regions, iii) living in 
a residential care home, and iv) having a history of previous hospitalizations. Clinical symptoms were collected according to the European CDC definition [9].

We included data and information about the underlying chronic medical conditions that increase the risk of severe SARS-COV-2 disease [9]. Specifically, they are diabetes, chronic kidney failure (CKF), hypertension, heart conditions and other cardiovascular and cerebrovascular diseases (CVD), chronic obstructive pulmonary disease (COPD), dyslipidaemia, autoimmune disease, neurologic conditions such as dementia [23] and mental disorders [24], glaucoma, asthma and cancer. In patients aged over 65 , a multidimensional geriatric examination, including a cognitive and functional assessment, was performed to assess dementia. Multimorbidity was defined as the coexistence of several conditions, without any of them taking predominance over the others. Accordingly, we defined multimorbidity as two or more chronic diseases in the patient [25].

The treatment data collection included: antivirals, hydroxychloroquine, tocilizumab or immunomodulatory and other adjunctive therapies for COVID-19 such as oxygen support, antibiotic drugs, steroid treatment and enoxaparin prophylaxis and treatment. The treatment prescription was based on national guidelines in use at enrolment time and validated by the WHO [26].

All data were checked by two physicians (DD and $\mathrm{DB}$ ), and a third physician (PD) decided upon any interpreting differences between the two primary reviewers. Because of clinicians' high workload, three medical statisticians from the University of Palermo performed raw data extraction, recording and data analysis (DM, LM and ME).

\section{Statistical analysis}

Categorical variables were summarized as counts and percentages, discrete and continuous variables as medians and interquartile range $(\mathrm{IQR})$. In the presence of an asymmetric distribution, quantitative variables were log-transformed. Pearson's correlations were calculated between pairs of quantitative variables. The differences between non-survivors and survivors were assessed through the Kaplan-Meier method and the proportional hazards (PH) Cox model. The PH Cox model was implemented using the "survival" package in the R environment (version 3.5.3). Due to the longitudinal nature of the data and the presence of time-varying variables, the time-dependent data set was built up according to the time-interval format [27], and the "coxph" function was used to estimate the parameters. This model at each event time compares the current covariate values of the subject who had the event to the current values of all others who were at risk at that time. Explanatory significant variables at univariable analysis were chosen as candidate prognostic factors for multivariable analysis. Moreover, a maximum of four explanatory variables was included to avoid overfitting. Moreover, variables containing more than $5 \%$ missing values were not considered in multivariable analysis. ROC-based threshold analysis was used to calculate the optimal cut-off value for laboratory findings, when appropriate. The cutoff value for statistical significance was a $p$-value $<0.05$. Clinical prognostic factors were included in multivariable analysis also with borderline $p$-values. The R script is available on the GitHub platform.

\section{Results}

\section{Sample characteristics}

From the beginning of March, there were 47 patients followed up during a 87 days observation period, accounting for a total of 1,035 person days. At the end of follow-up, 28 (60\%) patients were discharged, and 19 (40\%) died, so the estimated incidence density rate was 0.018 deaths per day (18 SARS-CoV-2-related deaths per 1,000 patient days). Six (13\%) patients were admitted to the ICU, and three of them died. Three (6\%) patients died within $24 \mathrm{~h}$ from admission.

The patients' median age was 75 (IQR: 59.5086.00). There were 24 (51\%) male patients.

The sample included a couple returning from a journey abroad (4\%), 12 (25\%) patients reported a history of previous hospitalization, 7 (15\%) came from a highly specialized hospital for cardiac surgery, and 15 (32\%) were from a residential care home.

The median length of stay was 18 days (IQR: 10.50-34.50), 12 days (IQR: 7-19.50) for non-survivors and 26.50 days (IQR: 11.75-36.25) for survivors.

\section{Comorbidities, symptoms and related therapies, and laboratory findings at hospital admission}

The recorded underlying medical conditions included 32 (68\%) patients with hypertension, $22(47 \%)$ patients with CVD, 10 (21\%) patients with dementia, 9 (19\%) were patients affected by type 2 diabetes, 9 (19\%) by CKF and 6 (12\%) patients with a mental disorder. Four (8\%) patients had COPD. Most patients, 33 (70\%), presented multimorbidity. There were $5(11 \%)$ patients presenting both diabetes and CVD, patients affected by dementia and CVD numbered 8 (17\%), while 2 (4\%) patients had all of these three diseases. Among non-survivors, 13 (68\%) had hypertension, 11 (58\%) had CVD, 7 (37\%) had CKF, 7 (37\%) had dementia, and 5 (26\%) had diabetes (Table I). 
Longitudinal investigation of severe acute respiratory syndrome coronavirus 2 (SARS-CoV-2) infection in older patients in the province of Palermo (Southern Italy) during the early wave of the pandemic

Table I. Demographic and clinical characteristics at hospital admission: HRs and 95\% Cls from univariate PH Cox model

\begin{tabular}{|c|c|c|c|c|c|c|}
\hline Variables & $\begin{array}{l}\text { Total sample } \\
\quad(n=47)\end{array}$ & $\begin{array}{l}\text { Status } \\
\text { survivor } \\
(n=28)\end{array}$ & $\begin{array}{c}\text { Status } \\
\text { non-survivor } \\
(n=19)\end{array}$ & HR & $95 \% \mathrm{Cl}$ & $P$-value \\
\hline Age, median (IQR) [years] & $75(59.50-86.00)$ & $64(53.25-78.50)$ & $85(76.50-88.50)$ & 1.05 & $1.01-1.10$ & 0.006 \\
\hline Sex, M & $24(51 \%)$ & $13(46 \%)$ & $11(58 \%)$ & 1.41 & $0.56-3.51$ & 0.459 \\
\hline Trip aboard, Yes & $2(4 \%)$ & $1(4 \%)$ & $1(5 \%)$ & 0.82 & $0.10-6.51$ & 0.851 \\
\hline Diabetes, Yes & $9(19 \%)$ & $4(14 \%)$ & $5(26 \%)$ & 3.48 & $1.17-10.37$ & 0.025 \\
\hline CKF, Yes & $9(19 \%)$ & $2(7 \%)$ & $7(37 \%)$ & 2.87 & $1.02-8.05$ & 0.046 \\
\hline Hypertension, Yes & $32(68 \%)$ & $19(68 \%)$ & $13(68 \%)$ & 1.2 & $0.38-3.73$ & 0.758 \\
\hline Cardiovascular disease, Yes & $22(47 \%)$ & $11(39 \%)$ & $11(58 \%)$ & 1.67 & $0.61-4.55$ & 0.314 \\
\hline COPD, Yes & $4(8 \%)$ & $1(4 \%)$ & $3(16 \%)$ & 1.43 & $0.39-5.28$ & 0.587 \\
\hline Dyslipidaemia, Yes & $6(13 \%)$ & $6(21 \%)$ & $0(0 \%)$ & n.a. & n.a. & n.a. \\
\hline Autoimmune disease, Yes & $4(8 \%)$ & $2(7 \%)$ & $2(11 \%)$ & 1.61 & $0.36-7.12$ & 0.529 \\
\hline Dementia, Yes & $10(21 \%)$ & $3(11 \%)$ & $7(37 \%)$ & 2.27 & $0.86-6.00$ & 0.097 \\
\hline Mental disease, Yes & $6(12 \%)$ & $4(14 \%)$ & $2(11 \%)$ & 0.65 & $0.14-2.95$ & 0.581 \\
\hline Multimorbidity, Yes & $33(70 \%)$ & $19(68 \%)$ & $14(74 \%)$ & 1.63 & $0.36-7.34$ & 0.521 \\
\hline Medical complications, Yes & $18(38 \%)$ & $11(39 \%)$ & $7(37 \%)$ & 0.79 & $0.27-2.32$ & 0.663 \\
\hline Glaucoma, Yes & $3(6 \%)$ & $2(7 \%)$ & $1(5 \%)$ & 0.94 & $0.12-7.17$ & 0.951 \\
\hline Asthma, Yes & $1(2 \%)$ & $1(4 \%)$ & $0(0 \%)$ & n.a. & n.a. & n.a. \\
\hline Thyroid, Yes & $6(13 \%)$ & $5(18 \%)$ & $1(5 \%)$ & 0.33 & $0.04-2.55$ & 0.29 \\
\hline Cancer disease, Yes & $2(4 \%)$ & $0(0 \%)$ & $2(11 \%)$ & 16.23 & $2.92-90.08$ & 0.001 \\
\hline $\begin{array}{l}\text { Polypharmacological } \\
\text { therapy, Yes }\end{array}$ & $21(45 \%)$ & $14(50 \%)$ & $7(37 \%)$ & 1.48 & $0.18-12.4$ & 0.72 \\
\hline Residential care homes, Yes & $15(32 \%)$ & $6(21 \%)$ & $9(47 \%)$ & 1.53 & $0.62-3.77$ & 0.359 \\
\hline Intensive care, Yes & $6(12 \%)$ & $3(11 \%)$ & $3(16 \%)$ & 3.49 & $0.44-27.37$ & 0.235 \\
\hline Previous hospitalization, Yes & $12(25 \%)$ & $8(28 \%)$ & $4(21 \%)$ & 0.49 & $0.16-1.49$ & 0.208 \\
\hline $\begin{array}{l}\text { Length of stay, median (IQR) } \\
\text { [days] }\end{array}$ & $18(10.50-34.50)$ & $26.50(11.75-36.25)$ & $12(7-19.50)$ & & & \\
\hline
\end{tabular}

Table II. Symptoms and related therapies at hospital admission: HRs and 95\% Cls from univariate PH Cox model

\begin{tabular}{|lcccccc|}
\hline Variables & $\begin{array}{c}\text { Total sample } \\
(n=47)\end{array}$ & $\begin{array}{c}\text { Status } \\
\text { survivor } \\
(n=28)\end{array}$ & $\begin{array}{c}\text { Status } \\
\text { non-survivor } \\
(n=19)\end{array}$ & HR & 95\% Cl & P-value \\
\hline Diarrhoea, Yes & $2(4 \%)$ & $2(7 \%)$ & $0(0 \%)$ & n.a. & n.a. & n.a. \\
\hline Fever, Yes & $20(43 \%)$ & $15(54 \%)$ & $5(26 \%)$ & 0.61 & $0.21-1.77$ & 0.364 \\
\hline Conjunctivitis, Yes & $1(2 \%)$ & $0(0 \%)$ & $1(5 \%)$ & 14.15 & $1.47-136.1$ & 0.022 \\
\hline Cough, Yes & $16(34 \%)$ & $11(39 \%)$ & $5(26 \%)$ & 0.79 & $0.27-2.32$ & 0.666 \\
\hline Arthromyalgia, Yes & $0(0 \%)$ & $0(0 \%)$ & $0(0 \%)$ & n.a. & n.a. & n.a. \\
\hline Asthenia, Yes & $5(11 \%)$ & $4(14 \%)$ & $1(5 \%)$ & 0.64 & $0.08-4.97$ & 0.673 \\
\hline Dyspnoea, Yes & $22(47 \%)$ & $14(50 \%)$ & $8(42 \%)$ & 1.11 & $0.39-3.18$ & 0.844 \\
\hline Ratio PaO, $/$ FIO ${ }_{2}, \leq 200$ & $25(53 \%)$ & $19(68 \%)$ & $6(32 \%)$ & 1.64 & $0.61-4.38$ & 0.325 \\
\hline Enoxaparin, Yes & $46(98 \%)$ & $28(100 \%)$ & $18(95 \%)$ & 0.77 & $0.17-3.41$ & 0.734 \\
\hline Hydroxychloroquine, Yes & $32(68 \%)$ & $22(79 \%)$ & $10(53 \%)$ & 0.41 & $0.15-1.10$ & 0.077 \\
\hline Steroids, Yes & $45(96 \%)$ & $27(96 \%)$ & $18(95 \%)$ & n.a. & n.a. & n.a. \\
\hline Antibiotics, Yes & $41(87 \%)$ & $27(96 \%)$ & $14(74 \%)$ & 0.30 & $0.09-0.95$ & 0.040 \\
\hline Ceftriaxone, Yes & $27(57 \%)$ & $18(64 \%)$ & $9(47 \%)$ & 0.93 & $0.37-2.36$ & 0.878 \\
\hline Macrolide, Yes & $20(42 \%)$ & $14(50 \%)$ & $6(32 \%)$ & 1.04 & $0.37-2.90$ & 0.940 \\
\hline Other antibiotic, Yes & $13(28 \%)$ & $8(29 \%)$ & $5(26 \%)$ & 0.41 & $0.12-1.44$ & 0.164 \\
\hline Tocilizumab, Yes & $5(11 \%)$ & $3(11 \%)$ & $2(11 \%)$ & 0.87 & $0.20-3.82$ & 0.854 \\
\hline
\end{tabular}

n.a. - not applicable, because there are no non-survivors. 
The most common symptoms were dyspnoea (47\%), fever (43\%), and cough (34\%). Almost all patients were treated with enoxaparin (98\%), steroids (96\%), and antibiotics (87\%), 32 (68\%) received hydroxychloroquine and 5 (11\%) tocilizumab (Table II). The median $\mathrm{PaO}_{2} / \mathrm{FlO}_{2}$ was $259 \mathrm{~mm} \mathrm{Hg}$ (IQR: 180-380) for the whole sample. It was $207.5 \mathrm{~mm} \mathrm{Hg}$ (IQR: 137.5-264.2) for non-survivors and $320 \mathrm{~mm} \mathrm{Hg}$ (IQR: 225-436) for survivors. The median $\mathrm{PaO}_{2} / \mathrm{FIO}_{2}$ was 280 (IQR: 230-321) for patients affected by dementia.

Among the hematologic parameters, the median value for neutrophil/lymphocyte ratio (NLR) was 2.90 (IQR: 1.99-5.57) with lower values for survivors (median $=2.63$, IQR: 1.95-4.25) than non-survivors (median $=3.21$, IQR: 2.68-8.24). Related blood coagulation, the median value for D-dimer was $509 \mu \mathrm{g}$ (IQR: 339-1227), and it was higher for non-survivors (median $=1515 \mu \mathrm{g}$, IQR: 532-2314) than for survivors (median $=483 \mu \mathrm{g}$ IQR: 331-920) (Table III). The day-to-day NLR was on average higher for non-survivors than survivors from the early stage of the disease to discharge/ death (Figure 1 A). A similar trend occurred for D-dimer, within the first 20 days of hospitalization (Figure $1 \mathrm{~B}$ ). The cut-off value for NLR was 7 (Se $=72 \%$; $\mathrm{Sp}=83 \%$ ) and for D-dimer was 855 (Se = $78 \% ; \mathrm{Sp}=63 \%)$.

\section{Univariable analysis}

There was an increased hazard for age $(H R=$ $1.05,95 \% \mathrm{Cl}: 1.01-1.10)$, diabetes $(\mathrm{HR}=3.48$, 95\% Cl: $1.17-10.37)$, CKF (HR $=2.87,95 \% \mathrm{Cl}: 1.02-$ $8.05)$ and cancer disease $(\mathrm{HR}=16.23,95 \% \mathrm{Cl}$ : 2.92-90.08) (Table I). Antibiotic therapy was pro-

Table III. Laboratory findings at hospital admission and day-to-day: HRs and 95\% Cls from univariate PH Cox Model

\begin{tabular}{|c|c|c|c|c|c|c|c|c|c|}
\hline \multirow[t]{2}{*}{ Variables } & \multirow{2}{*}{$\begin{array}{l}\text { Total sample } \\
\quad(n=47)\end{array}$} & \multirow{2}{*}{$\begin{array}{l}\text { Status } \\
\text { survivor } \\
(n=28)\end{array}$} & \multirow{2}{*}{$\begin{array}{c}\text { Status } \\
\text { non-survivor } \\
(n=19)\end{array}$} & \multicolumn{3}{|c|}{ At hospital admission } & \multicolumn{3}{|c|}{ Day-to-day } \\
\hline & & & & $H R^{\S}$ & $95 \% \mathrm{Cl}$ & $P$-value & $H R^{\S}$ & $95 \% \mathrm{Cl}$ & $P$-value \\
\hline $\mathrm{Hb}$ & $\begin{array}{c}12.05 \\
(10.95-13.55)\end{array}$ & $\begin{array}{c}12.05 \\
(10.28-13.22)\end{array}$ & $\begin{array}{c}12.10 \\
(11.32-14.45)\end{array}$ & 1.18 & $0.93-1.50$ & 0.163 & 0.96 & $0.71-1.29$ & 0.772 \\
\hline $\begin{array}{l}\text { Eosinophil } \\
\text { count }[\times 10 / I]\end{array}$ & $\begin{array}{c}10 \\
(0-60)\end{array}$ & $\begin{array}{c}15 \\
(6.75-105)\end{array}$ & $\begin{array}{c}0 \\
(0-10)\end{array}$ & 0.86 & $0.67-1.09$ & 0.207 & 0.63 & $0.47-0.83$ & 0.001 \\
\hline $\begin{array}{l}\text { Lymphocyte } \\
\text { count }[\times 10 / 1]\end{array}$ & $\begin{array}{c}1360 \\
(930-1620)\end{array}$ & $\begin{array}{c}1370 \\
(1125-1545) \\
\end{array}$ & $\begin{array}{c}1020 \\
(790-1770)\end{array}$ & 0.60 & $0.21-1.71$ & 0.340 & 0.30 & $0.15-0.58$ & $<0.001$ \\
\hline $\begin{array}{l}\text { Neutrophil } \\
\text { count }[\times 10 / I]\end{array}$ & $\begin{array}{c}3745 \\
(2718-5858) \\
\end{array}$ & $\begin{array}{c}3435 \\
(2652-5155) \\
\end{array}$ & $\begin{array}{c}5030 \\
(3305-9008) \\
\end{array}$ & 1.99 & $0.84-4.75$ & 0.119 & 2.20 & $1.12-4.33$ & 0.023 \\
\hline $\begin{array}{l}\text { Monocyte } \\
\text { count }[\times 10 / I]\end{array}$ & $\begin{array}{c}545 \\
(390-772)\end{array}$ & $\begin{array}{c}460 \\
(405-780)\end{array}$ & $\begin{array}{c}570 \\
(370-770)\end{array}$ & 1.05 & $0.63-1.78$ & 0.840 & 0.48 & $0.30-0.77$ & 0.002 \\
\hline$N L R>7$ & $\begin{array}{c}9 \\
(19 \%)\end{array}$ & $\begin{array}{c}3 \\
(11 \%)\end{array}$ & $\begin{array}{c}6 \\
(32 \%)\end{array}$ & 2.52 & $0.90-7.05$ & 0.079 & 12.69 & $2.82-57.18$ & $<0.001$ \\
\hline $\begin{array}{l}\text { Platelet count } \\
{[\times 10 / I]}\end{array}$ & $\begin{array}{c}198 \\
(161.5-299) \\
\end{array}$ & $\begin{array}{c}219 \\
(163.5-299) \\
\end{array}$ & $\begin{array}{c}193 \\
(153.5-268.75) \\
\end{array}$ & 0.79 & $0.32-1.92$ & 0.599 & 0.88 & $0.67-1.15$ & 0.349 \\
\hline Ferritin [ $\mathrm{\mu g} / \mathrm{l}]$ & $\begin{array}{c}345 \\
(190.2-693.5) \\
\end{array}$ & $\begin{array}{c}277 \\
(153.5-590) \\
\end{array}$ & $\begin{array}{c}590 \\
(399-1303) \\
\end{array}$ & 1.46 & $0.72-2.95$ & 0.294 & 0.66 & $0.08-5.36$ & 0.701 \\
\hline $\mathrm{LDH}[\mathrm{mU} / \mathrm{ml}]$ & $\begin{array}{c}237 \\
(191.5-291) \\
\end{array}$ & $\begin{array}{c}220 \\
(158-262) \\
\end{array}$ & $\begin{array}{c}268 \\
(232-760) \\
\end{array}$ & 2.90 & $1.29-6.56$ & 0.010 & 5.10 & $1.33-19.54$ & 0.017 \\
\hline D-dimer $[\mu \mathrm{g}]$ & $\begin{array}{c}509 \\
(339-1227) \\
\end{array}$ & $\begin{array}{c}483 \\
(331-920)\end{array}$ & $\begin{array}{c}1515 \\
(532-2314)\end{array}$ & 2.67 & $1.08-6.60$ & 0.034 & 3.43 & $1.60-7.36$ & 0.002 \\
\hline $\begin{array}{l}\text { C-reactive } \\
\text { protein } \\
{[\mathrm{mg} / \mathrm{dl}]}\end{array}$ & $\begin{array}{c}3.38 \\
(1.10-8.95)\end{array}$ & $\begin{array}{c}2.45 \\
(0.85-5.84)\end{array}$ & $\begin{array}{c}7.13 \\
(2.94-19.39)\end{array}$ & 1.07 & $1.03-1.12$ & $<0.001$ & 1.09 & $1.05-1.13$ & $<0.001$ \\
\hline $\begin{array}{l}\text { Creatinine } \\
{[\mathrm{mg} / \mathrm{dl}]}\end{array}$ & $\begin{array}{c}0.92 \\
(0.67-1.09) \\
\end{array}$ & $\begin{array}{c}0.80 \\
(0.65-1.03) \\
\end{array}$ & $\begin{array}{c}1.05 \\
(0.83-1.65) \\
\end{array}$ & 11.97 & $2.63-54.52$ & 0.001 & 2.70 & $1.43-5.09$ & 0.002 \\
\hline $\begin{array}{l}\text { Bilirubin } \\
{[\mathrm{mg} / \mathrm{dl}]}\end{array}$ & $\begin{array}{c}0.60 \\
(0.40-0.99)\end{array}$ & $\begin{array}{c}0.60 \\
(0.50-0.87)\end{array}$ & $\begin{array}{c}0.55 \\
(0.37-1.10)\end{array}$ & 1.03 & $0.99-1.08$ & 0.174 & 0.98 & $0.72-1.35$ & 0.927 \\
\hline $\begin{array}{l}\text { Troponin } \\
{[\mathrm{mg} / \mathrm{dl}]}\end{array}$ & $\begin{array}{c}7.80 \\
(2.35-14.85) \\
\end{array}$ & $\begin{array}{c}5.6 \\
(1.4-12) \\
\end{array}$ & $\begin{array}{c}15.65 \\
(12.38-24.10) \\
\end{array}$ & 1.12 & $0.77-1.62$ & 0.545 & 1.24 & $0.47-3.32$ & 0.663 \\
\hline GOT [mU/ml] & $\begin{array}{c}30.5 \\
(20.75-49) \\
\end{array}$ & $\begin{array}{c}27.5 \\
(20.25-36.75) \\
\end{array}$ & $\begin{array}{c}45 \\
(28.75-58) \\
\end{array}$ & 1.01 & $1.00-1.12$ & 0.014 & 1.01 & $1.00-1.01$ & 0.201 \\
\hline GPT [mU/ml] & $\begin{array}{c}19.0 \\
(11-31.50)\end{array}$ & $\begin{array}{c}23 \\
(11.50-29.25)\end{array}$ & $\begin{array}{c}19 \\
(11-31.5)\end{array}$ & 1.01 & $0.99-1.02$ & 0.276 & 0.98 & $0.95-1.01$ & 0.176 \\
\hline
\end{tabular}

\$The following laboratory findings were log-transformed before PH Cox model: eosinophils, lymphocytes, neutrophils, monocytes, platelets, ferritin, LDH, D-dimer, troponin. 
A

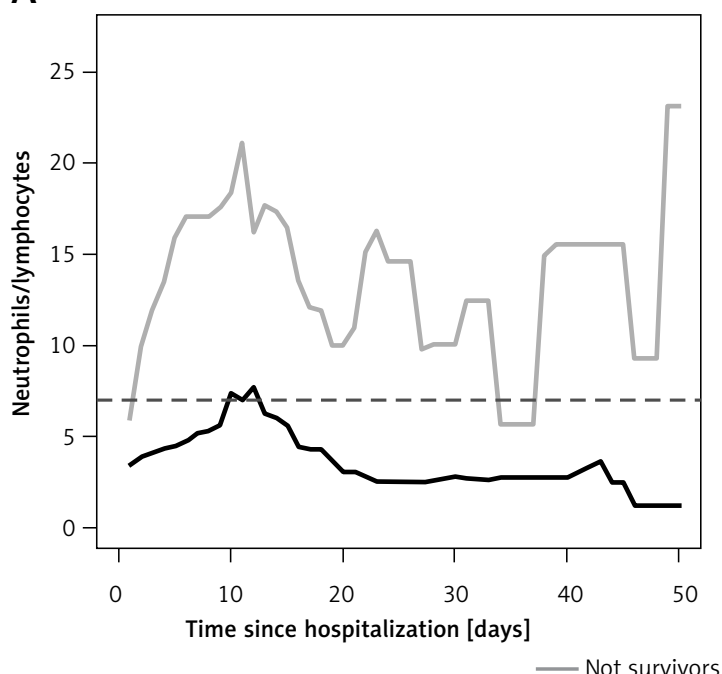

B

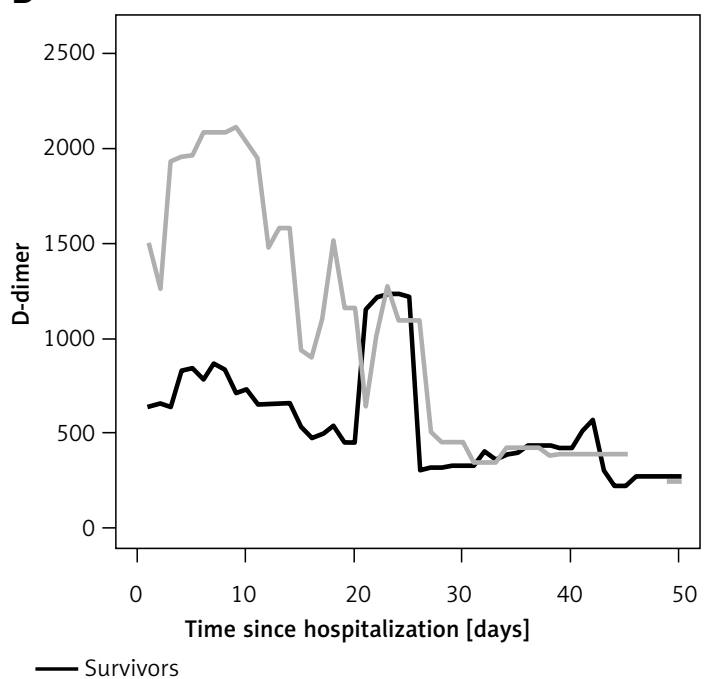

Figure 1. Comparison between survivors and non-survivors in terms of day-to-day mean NLR variation, with horizontal line at $\mathrm{NLR}=7$ (A) and mean D-dimer variation (B)

tective (HR =0.30, 95\% Cl: 0.09-0.95) (Table II). Regarding day-to-day laboratory findings, there was a significant hazard for $\log (\mathrm{LDH})(\mathrm{HR}=5.10$, 95\% Cl: 1.33-19.54), $\log (\mathrm{D}$-dimer $)(H R=3.43$, $95 \% \mathrm{Cl}: 1.60-7.36)$, C-reactive protein $(H R=1.09$, $95 \% \mathrm{Cl}: 1.05-1.13)$ and creatinine $(\mathrm{HR}=2.70$, $95 \% \mathrm{Cl}: 1.43-5.09)$. Patients with NLR $>7$ showed a significantly increased hazard $(H R=12.69$, 95\% Cl: 2.82-57.18) (Table III).

\section{Multivariable analysis}

At multivariable analysis, there was a significant hazard for diabetes $(\mathrm{HR}=8.13,95 \% \mathrm{Cl}$ : $1.91-$ 34.67), CKF ( $\mathrm{HR}=5.86,95 \% \mathrm{Cl}: 1.36-25.21)$, dementia ( $\mathrm{HR}=7.84,95 \% \mathrm{Cl}: 1.80-34.20)$, and NLR $>7$ ( $\mathrm{HR}=10.37,95 \% \mathrm{Cl}: 2.24-48.14)$ (Table IV). The model's predictive performance was already satisfactory on the third day of follow-up (AUC $\geq 0.0 .905$ ) (Figure 2).

\section{Discussion}

In the present study, we described the epidemiological and clinical prognostic factors, therapy and clinical course of SARS-CoV-2 infection in old adult patients, taking full advantage of the longitudinal cohort study design. In this way, we used the day-to-day follow-up to detect some significant prognostic factors of SARS-CoV-2 infection in the province of Palermo at the early stage of the pandemic.

Our sample's crude fatality rate was 40\% (19 out of 47 patients) in a window of time of 87 days. The comparison with other clinical studies is not so immediate, due to heterogeneous study design and patients' enrolment in the literature. Colaneri et al. [13] estimated a case fatality rate of $4.5 \%$ ( 2 deaths) in a sample of 44 patients enrolled be- tween 21 and 28 February 2020, 57\% of whom were over 65 . Limiting our observation to a similar time-span, the estimated case fatality rate in our sample in the first week of enrolment was $10.6 \%$ (5 deaths), and the percentage of people aged $65+$ was $68 \%$. Bruno et al. [5] estimated a case fatality rate of $19 \%$ ( 6 deaths) in a sample of 31 patients enrolled between 25 February and 29 April 2020, all aged $65+$. Of note, mortality for SARS-CoV-2 is age-dependent with the highest frequencies observed in young-old (approximately 65-74), middle-old (ages 75-84), and old-old (over age 85) people, the three life-stage subgroups of the older adult population [9].

The median length of hospital stay of survivors in our study was 26 days (Table I), comparable to 23 days reported by Bruno et al. [5] in patients aged 75 years or older admitted to an infectious diseases unit in Southern Italy.

In multivariable analysis, diabetes, dementia and CKF were significantly associated with mortality (Table IV). Diabetes is a known prognostic factor for the progression and mortality for SARSCoV-2 infection, due to its chronic inflammatory state that causes an imbalance of immune system response. Moreover, SARS-CoV-2 infection is a virus-induced systemic disease characterized by

Table IV. Clinical characteristics and day-to-day laboratory findings: HRs and $95 \% \mathrm{Cls}$ from multivariate $\mathrm{PH}$ Cox model

\begin{tabular}{|lccc|}
\hline Variables $^{\S}$ & HR & $95 \%$ Cl & $P$-value \\
\hline Diabetes, Yes & 8.13 & $1.91-34.67$ & 0.005 \\
\hline CKF, Yes & 5.86 & $1.36-25.21$ & 0.017 \\
\hline Dementia, Yes & 7.84 & $1.80-34.20$ & 0.006 \\
\hline NLR $>$ 7, Yes & 10.37 & $2.24-48.14$ & 0.003 \\
\hline
\end{tabular}

${ }^{\S}$ Only statistically significant covariates are in table. 
A

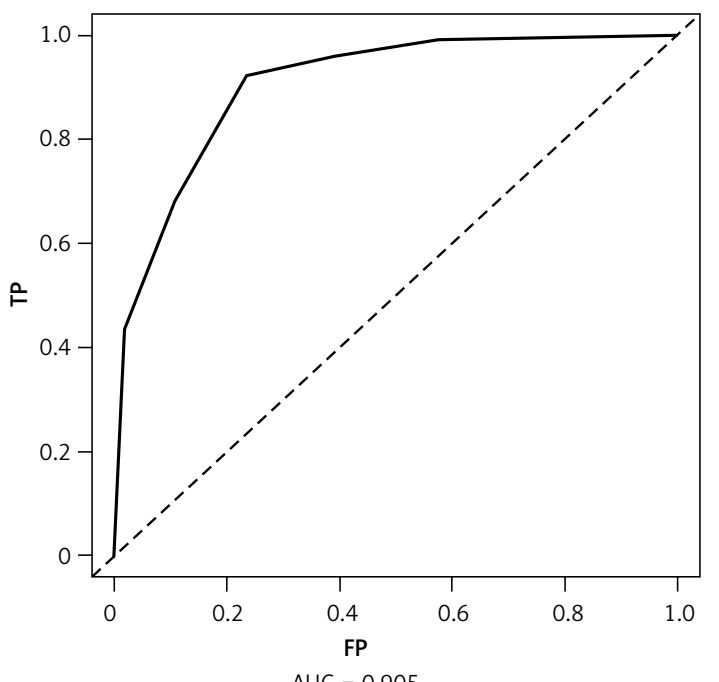

C

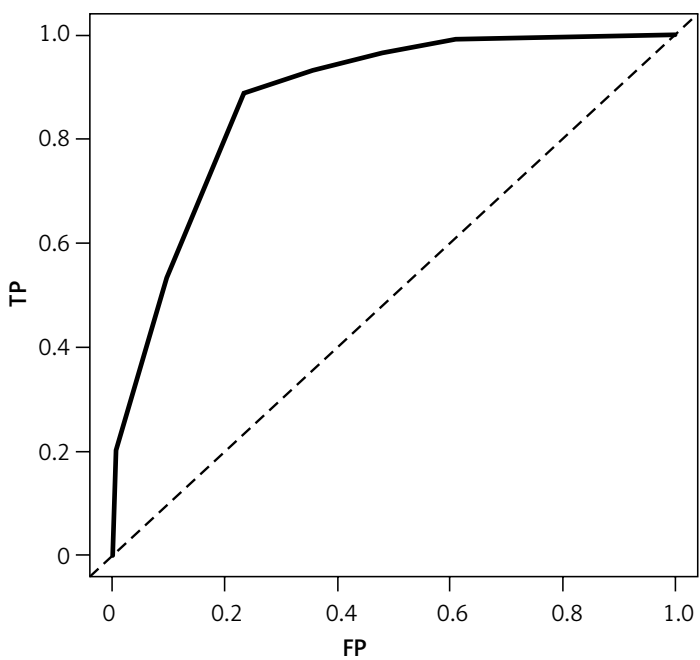

$\mathrm{AUC}=0.876$

E

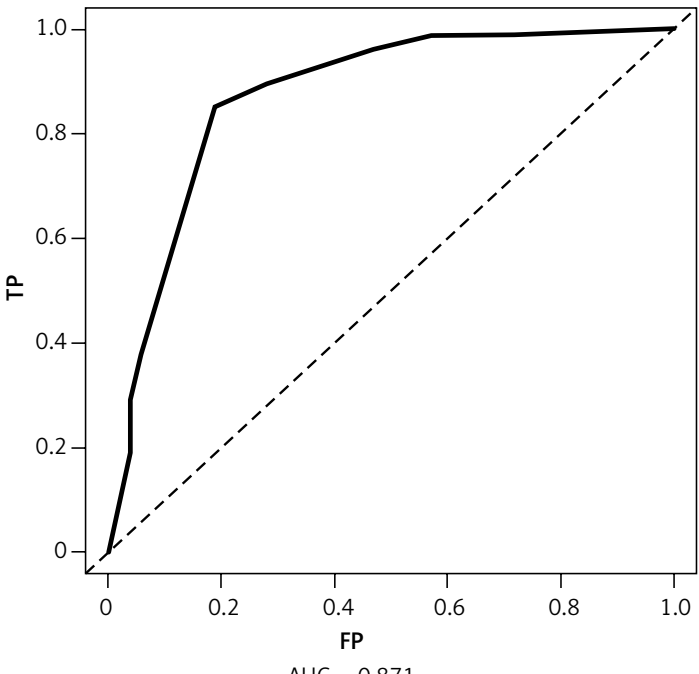

B

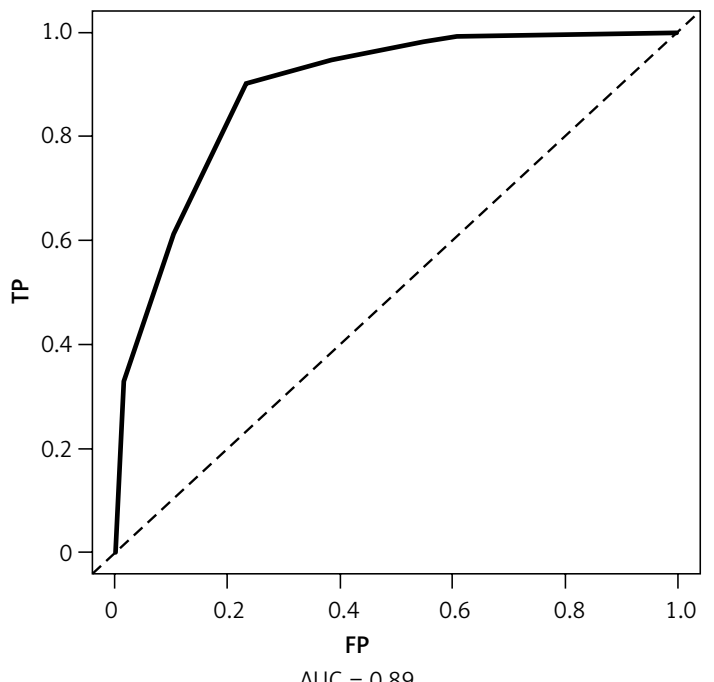

D

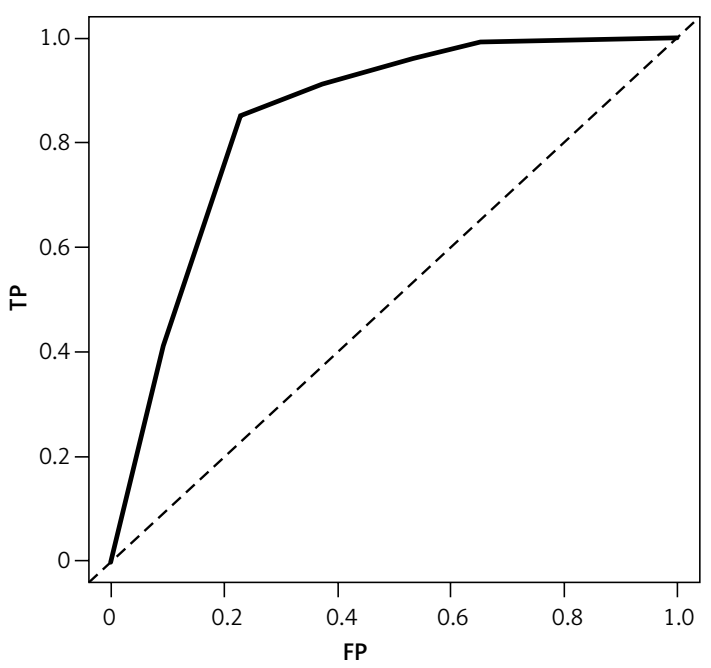

$\mathrm{AUC}=0.844$

$\mathrm{F}$

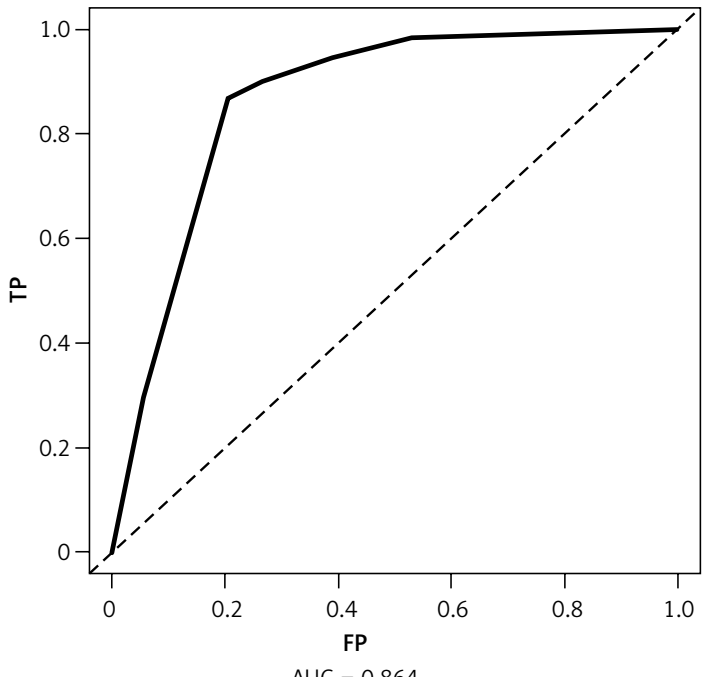

Figure 2. Predictive performance of the model: time-dependent ROC curves and predicted AUCs at times 3 (A), 5 (B), 7 (C), 14 (D), 21 (E), 28 (F) 
vasculitis and vasculopathy [28], which constitutes a red flag for diabetic patients with COVID-19. Furthermore, the study showed type 2 diabetes mellitus in association with obesity, one of the main prognostic factors for SARS-CoV-2 mortality [29].

Dementia is an emerging prognostic factor for the severe clinical course of the SARS-CoV-2 infection. In our study sample, dementia occurred in $21 \%$ of over 65 -year-old enrolled patients with a median age of 85.5 years and was associated with severe lung damage, as reported by the analysis of $\mathrm{PaO}_{2} / \mathrm{FIO}_{2}$ ratio. The lungs' imaging features performed during hospitalization supported the severity of pulmonary injury due to SARS-CoV-2 virus infection in dementia patients. Severe acute respiratory syndrome worsens with increasing age and in the presence of pre-existing dementia [30]. We found these patients with an atypical presentation, characterized by a few typical symptoms, specifically dyspnoea, fever and cough. In agreement with this result, Bianchetti et al. [30] found an atypical presentation for dementia patients with SARS-CoV-2 infection.

Prognosis of dementia patients worsens in the presence of both diabetes and hypertension, which are some of the most frequent underlying conditions reported in our study population. Nursing and clinical management of these subjects are challenging, so actions are desirable for implementing clinical knowledge and best practice for the early detection and management of hospitalized dementia patients with SARS-CoV-2 infection.

In our study, there was evidence of increased mortality risk for patients with CKF. Such patients have weaker immune systems, which can explain the increased risk of SARS-CoV-2 mortality by increasing the level of kidney dysfunction [31].

It is well known that NLR is a significant prognostic factor of respiratory and systemic infection $[32,33]$. Our study strengthens the role of NLR as a fast, simple and easily measured inflammatory biomarker [33]. Furthermore, we also observed elevated D-dimer levels for non-survivors (Figure 1). The association between increased D-dimers and severe SARS-CoV-2 infection may in part be explained by the interplay between inflammatory response and activation of coagulation. In SARS-CoV-2 patients, D-dimer level is included, especially in the early phase of the disease, as a test to detect severe complications such as deep vein thrombosis or pulmonary embolism (DVT/ $P E)$ [34]. The cut-off value of $500 \mu \mathrm{g} / \mathrm{l}$ is intended to exclude $P E$ in otherwise healthy patients [35]. However, the age-adjusted cut-off defined as age $\times 10$ was demonstrated to exclude PE in patients aged 50 years old or older $[35,36]$. In our study, the estimated cut-off value for D-dimer was found to be 855 , in line with the age-adjusted cutoff. In order to identify severe complications such as acute DVT/PE, D-dimer monitoring is advisable from the first week after symptoms' onset [37]. Since many SARS-CoV-2 patients showed underlying diseases that may trigger an increase in D-dimer levels, we suggest integrating D-dimer with other parameters such as NLR $>7$, the ratio $\mathrm{PaO}_{2} / \mathrm{FIO}_{2}$ and the occurrence of other underlying medical conditions, e.g. diabetes, dementia and CKF. We demonstrated the optimal prognostic value represented by the joint clinical evaluation of all these factors already in the first week of follow-up.

The day-to-day follow-up is the main strength of this study, which allowed the ongoing laboratory and clinical data collection, typical of the patient's initial classification and emergency context. Our study's main limitation regards the small sample size, which is, however, the total population referred to Partinico COVID Hospital and reflects the extent of the epidemic at the very early stage in the province of Palermo. For best data interpretation, it should be considered that the small sample size can affect the estimates' precision, as shown by wider confidence intervals. Secondly, missing data in many clinical and laboratory findings prevented us from including them in the statistical analysis.

Despite the evolution of sophisticated adjuncts to healthcare and the improvements of structured critical care systems, the multidisciplinary medical emergency approach and detailed and continuous data collection play a crucial role in detecting significant prognostic factors and improving SARSCoV-2 infection outcomes in old patients.

The in-depth knowledge of the complete clinical course of SARS-CoV-2 is essential in monitoring and treating the disease in a hospital setting and contributes to improving the patients' home management, especially older ones with frailty, such as those with dementia and/or diabetes. It has been shown that patients with dementia are at a higher risk of neuropsychiatric disorders due to social isolation and shelter-in-place orders $[38,39]$ and that SARS-CoV-2 patients affected by diabetes experience deterioration of lifestyle and disruption of their periodic monitoring [40]. Further research may arise from the cooperation with primary health care with the aim to monitor discharged patients and assess the long-term COVID19 impact.

\section{Acknowledgments}

We are grateful to Dr Salvatore Di Rosa, Dr Francesca Provenzano and Dr Vincenzo Provenzano of Partinico COVID Hospital for their valuable comments and relevant review of this manuscript. We are grateful to our linguistic proof-reader for improving the fluency and vocabulary of this man- 
uscript. We affirm that we have not received funds for conducting this research.

\section{Conflict of interest}

The authors declare no conflict of interest.

\section{References}

1. Istat, Istituto Superiore di Sanità, 2020, Impatto dell'Epidemia Covid-19 sulla mortalità: Cause di morte nei deceduti positivi a Sars-Cov-2, https://www.epicentro.iss. it/coronavirus/pdf/Rapp_Istat_Iss_9luglio.pdf, Accessed 9 November 2020

2. Grasselli G, Zangrillo A, Zanella A, et al. Baseline characteristics and outcomes of 1591 patients infected with SARS-CoV-2 admitted to ICUs of the Lombardy Region, Italy. JAMA 2020; 323: 1574-81.

3. Biagi A, Rossi L, Malagoli A, et al. Clinical and epidemiological characteristics of 320 deceased patients with COVID-19 in an Italian Province: a retrospective observational study. J Med Virol 2020; 92: 2718-24.

4. Pisapia R, Pisaturo M, Fusco FM, et al. Differences among confirmed and not-confirmed COVID-19 patients at "d.Cotugno" hospital, Naples (Italy): what we learned from first suspected cases? Infez Med 2020; 28 : 84-8.

5. Bruno G, Perelli S, Fabrizio C, Buccoliero GB. Short-term outcomes in individuals aged 75 or older with severe coronavirus disease (COVID-19): first observations from an infectious diseases unit in Southern Italy. J Infect 2020; 81: e86-8.

6. Saglietto A, D'Ascenzo F, Zoccai GB, De Ferrari GM COVID-19 in Europe: the Italian lesson. Lancet 2020; 395: 1110-1.

7. Roviello V, Roviello GN. Lower COVID-19 mortality in Italian forested areas suggests immunoprotection by Mediterranean plants. Environ Chem Lett 2020; 19: 699-710.

8. Bianconi V, Bronzo P, Banach M, Sahebkar A, Mannarino MR, Pirro $M$. Particulate matter pollution and the COVID-19 outbreak: results from Italian regions and provinces. Arch Med Sci 2020; 16: 985-92.

9. CDC 2020, Coronavirus (COVID-19), https://www.cdc. gov/coronavirus/2019-ncov/index.html, Accessed 9 November 2020

10. Yan X, Han X, Peng D, et al. Clinical characteristics and prognosis of 218 patients with COVID-19: a retrospective study based on clinical classification. Front Med 2020; 7: 485.

11. Chon YJ, Kim JY, Suh YJ, et al. Adverse initial CT findings associated with poor prognosis of coronavirus disease. J Korean Med Sci 2020; 35: e316.

12. Wu C, Chen X, Cai Y, et al. Risk factors associated with acute respiratory distress syndrome and death in patients with coronavirus disease 2019 pneumonia in Wuhan, China. JAMA Intern Med 2020; 180: 934-43.

13. Colaneri M, Sacchi P, Zuccaro V, et al. Clinical characteristics of coronavirus disease (COVID-19) early findings from a teaching hospital in Pavia, North Italy, 21 to 28 February 2020. Euro Surveill 2020; 25: 2000460.

14. De Rossi N, Scarpazza C, Filippini C, et al. Early use of low dose tocilizumab in patients with COVID-19: a retrospective cohort study with a complete follow-up. EClinicalMedicine 2020; 25: 100459.

15. Hwang J, Kim J, Park J, Chang MC, Park D. Neurological diseases as mortality predictive factors for patients with COVID-19: a retrospective cohort study. Neurol Sci 2020; 41: 2317-24.

16. Yan Y, Yang Y, Wang F, et al. Clinical characteristics and outcomes of patients with severe covid-19 with diabetes. BMJ Open Diabetes Res Care 2020; 8: e001343.

17. Liu J, Zhang S, Wu Z, et al. Clinical outcomes of COVID-19 in Wuhan, China: a large cohort study. Ann Intensive Care 2020; 10: 99.

18. Naymagon L, Zubizarreta N, Feld J, et al. Admission D-dimer levels, D-dimer trends, and outcomes in COVID-19. Thromb Res 2020; 196: 99-105.

19. Manson JJ, Crooks C, Naja M, et al. COVID-19-associated hyperinflammation and escalation of patient care: a retrospective longitudinal cohort study. Lancet Rheumat 2020; 2: e594-602.

20. Chen R, Sang L, Jiang M, et al. Longitudinal hematologic and immunologic variations associated with the progression of COVID-19 patients in China. J Allergy Clin Immunol 2020; 146: 89-100.

21. Zhou F, Yu T, Du R, et al. Clinical course and risk factors for mortality of adult inpatients with COVID-19 in Wuhan, China: a retrospective cohort study. Lancet 2020; 395: 1054-62.

22. Corman VM, Landt O, Kaiser M, et al. Detection of 2019 novel coronavirus (2019-nCoV) by real-time RT-PCR. Euro Surveill 2020; 25: 2000045.

23. Hughes CP, Berg L, Danziger WL, Coben LA, Martin RL. A new clinical scale for the staging of dementia. Br J Psychiatry 1982; 140: 566-72.

24. WHO, 2020, Mental disorders, https://www.who.int/ news-room/fact-sheets/detail/mental-disorders, Accessed 9 November 2020.

25. Johnston MC, Crilly M, Black C, Prescott GJ, Mercer SW, Defining and measuring multimorbidity: a systematic review of systematic reviews. Eur J Public Health 2019; 29: 182-9.

26. WHO, 2020, Clinical management of COVID-19, Internim guidance 27 May 2020, https://apps.who.int/iris/bitstream/ handle/10665/332196/WHO-2019-nCoV-clinical-2020. 5-eng.pdf?sequence $=1 \&$ isAllowed $=y$, Accessed 9 November 2020.

27. Thernau T, Crowson C, Atkinson E. Using Time Dependent Covariates and Time Dependent Coefficients in the Cox Model 2020. https://cran.r-project.org/web/packages/survival/vignettes/timedep.pdf. Accessed 9 November 2020.

28. Becker RC. COVID-19-associated vasculitis and vasculopathy. J Thromb Thrombolysis 2020; 50: 499-511.

29. Roncon L, Zuin M, Rigatelli G, Zuliani G. Diabetic patients with COVID-19 infection are at higher risk of ICU admission and poor short-term outcome. J Clin Virol 2020; 127: 104354.

30. Bianchetti A, Rozzini R, Guerini F, et al. Clinical presentation of COVID19 in dementia patients. J Nutr Health Aging 2020; 24: 560-2.

31. Williamson EJ, Walker AJ, Bhaskaran K, et al. Factors associated with COVID-19-related death using OpenSAFELY. Nature 2020; 584: 430-6.

32. Han $\mathrm{Q}$, Wen $X$, Wang L, et al. Role of hematological parameters in the diagnosis of influenza virus infection in patients with respiratory tract infection symptoms. J Clin Lab Anal 2020; 34: e23191.

33. Liu L, Zheng Y, Cai L, et al. Neutrophil-to-lymphocyte ratio, a critical predictor for assessment of disease severity in patients with COVID-19. Int J Lab Hematol 2020; https://doi.org/10.1111/ijlh.13374. 
34. Rostami M, Mansouritorghabeh H. D-dimer level in COVID-19 infection: a systematic review. Exp Rev Hematol 2020; 13: 1265-75.

35. Douma RA, Le Gal G, Söhne M, et al. Potential of an age adjusted D-dimer cut-off value to improve the exclusion of pulmonary embolism in older patients: a retrospective analysis of three large cohorts. BMJ 2010; 340: c1475.

36. Righini M, Van Es J, Den Exter PL, et al. Age-adjusted D-dimer cutoff levels to rule out pulmonary embolism: the ADJUST-PE study. JAMA 2014; 311: 1117-24.

37. Goldin Y, Pasvolsky O, Rogowski O, et al. The diagnostic yield of D-Dimer in relation to time from symptom onset in patients evaluated for venous thromboembolism in the emergency medicine department. J Thrombosis Thrombol 2011; 31: 1-5.

38. Numbers K, Brodaty $\mathrm{H}$. The effects of the COVID-19 pandemic on people with dementia. Nature Rev Neurol 2021; 17: 69-70.

39. Miceli S, Maniscalco L, Matranga D. Social networks and social activities promote cognitive functioning in both concurrent and prospective time: evidence from the SHARE survey. Eur J Ageing 2019; 16: 145-54.

40. Ng KE. The effect of COVID-19 on patients with diabetes. US Pharm 2020; 45: 9-12. 